\title{
Ultimate and proximate mechanisms of reciprocal altruism in rats
}

\author{
Vassilissa Dolivo $^{1} \cdot$ Claudia Rutte $^{2} \cdot$ Michael Taborsky $^{1}$
}

Published online: 5 August 2016

(C) Psychonomic Society, Inc. 2016

\begin{abstract}
The reciprocal exchange of goods and services among social partners is a conundrum in evolutionary biology because of its proneness to cheating, but also the behavioral and cognitive mechanisms involved in such mutual cooperation are hotly debated. Extreme viewpoints range from the assumption that, at the proximate level, observed cases of "direct reciprocity" can be merely explained by basic instrumental and Pavlovian association processes, to the other extreme implying that "cultural factors" must be involved, as is often attributed to reciprocal cooperation among humans. Here we argue that neither one nor the other extreme conception is likely to explain proximate mechanisms underlying reciprocal altruism in animals. In particular, we outline that Pavlovian association processes are not sufficient to explain the documented reciprocal cooperation among Norway rats, as has been recently argued.
\end{abstract}

Keywords Cooperation · Direct reciprocity $\cdot$ Pavlovian conditioning $\cdot$ Cognition $\cdot$ Rattus norvegicus

In his paper "A Cognitive Behaviorist Approach to the Study of Animal Behavior," Thomas Zentall (2002) outlined that biologists and psychologists have very different perspectives when trying to understand animal behavior. Therefore, it seems useful to contrast different concepts and approaches

Vassilissa Dolivo

vassilissadolivo@hotmail.com

1 Behavioural Ecology, Institute of Ecology and Evolution, University of Bern, Wohlenstrasse 50a, CH-3032 Hinterkappelen, Switzerland

2 Swiss National Science Foundation, Wildhainweg 3, P.O. Box 8232, CH-3001 Bern, Switzerland in experimental studies of animal behavior. Obviously, these alternative approaches are not mutually exclusive but rather complementary. We thus welcome Zentall's (2016) discussion of our studies of reciprocal cooperation among Norway rats and respond here by taking the "biological," or evolutionary, perspective.

While studying modalities of cooperation among Norway rats, we found that Rattus norvegicus cooperates using direct reciprocity in an iterated prisoner's dilemma, helping preferentially cooperators instead of defectors (Rutte \& Taborsky 2008). More recently, in an experiment involving two conspecific cooperators providing test rats with "appreciated bananas" or "less appreciated carrots," we found that rats distinguish between different cooperators depending on the quality of their help (Dolivo \& Taborsky, 2015a). Test subjects provided more food to social partners that had previously provided them with the more appreciated food. The delay before reciprocating was also adjusted to the partner's perceived helping quality. When cooperating with two conspecific partners expending the same effort, rats apparently rely on obtained benefit to adjust their level of reciprocation. Zentall (2016) challenged our findings by arguing that rather than demonstrating "direct reciprocity," simple Pavlovian conditioning mechanisms could suffice to explain our observations. Here we explain why Pavlovian conditioning mechanisms alone cannot explain the rats' behavior in our experiments, and that the interpretation offered by Zentall (2016) does not do justice to the experimental evidence.

Zentall (2016) rightly emphasized that the ability to detect cheats is of crucial importance for the evolution of altruism based on direct reciprocity, and that this requires a certain capacity of individual recognition and memory. Recognition and long-term memory of individuals has been demonstrated in birds (Boeckle \& Bugnyar, 2012; Godard 1991) and mammals (Bruck, 2013; Kendrick, da Costa, Leigh, Hinton, 
\& Peirce, 2001), suggesting that this precondition for cooperation through direct reciprocity is met at least in these vertebrate taxa. Long-term memory has been demonstrated also in laboratory strains of Norway rats (Babb \& Crystal, 2006; Veyrac et al., 2015) and in a number of other vertebrates (see Zentall, 2013, for review), and even invertebrates such as bees or fruit flies are capable of several forms of learning and memory, with neuronal mechanisms of learning appearing to be homologous to ours (Davis, 2005; Kawecki, 2010; Mery \& Kawecki, 2002). Therefore, we think there is little reason to believe that reciprocal altruism can be expected only "in species closely related to us, like apes and perhaps monkeys" due to the limited cognitive abilities of other animals, as has been argued (Zentall, 2016). At variance with this assumption, contingent cooperation has been observed more often in nonprimates than in primates, which suggests that reciprocity among animals may not be severely constrained by cognitive ineptitude (Cheney, 2011). Many animals live in more or less stable and often multilevel societies (Cockburn, 1998; de Silva \& Wittemyer, 2012; Grueter, Matsuda, Zhang, \& Zinner, 2012; Jungwirth \& Taborsky, 2015; Riehl, 2013; Wright \& McDonald, 2016), which implies that individuals know and remember the ranks and roles of their social partners, thus involving individual recognition and the memory of previous interactions as well as their outcomes. This ability is sufficient to allow individuals to respond to previously received help of social partners, which should in fact enable a large range of animal taxa to cooperate among each other by applying the decision rule "help someone who has helped you before" (i.e., direct reciprocity; Taborsky, Frommen, \& Riehl, 2016, p. 3).

Zentall (2016) observed correctly that from an evolutionary perspective, reciprocal altruism can only be selected for if the cost of occasionally sharing one's resources with others is outweighed by the benefit of receiving resources from others. Recent findings, such as the reciprocal cooperation in vampire bats (Carter \& Wilkinson, 2013, 2015), reveal mechanisms maintaining cooperation based on a social network, which implies that either simple reciprocity rules (van Doorn \& Taborsky 2012) or more elaborate exchange mechanisms (Carter \& Wilkinson, 2015) might be commonly applied in species living in complex societies. It is a great challenge for future research to unravel which cognitive skills are required for reciprocal cooperation in species characterized by complex social network structures (Carter \& Wilkinson, 2015, 2016).

We are not convinced that genetic predispositions, basic learning processes (Pavlovian conditioning), and more complex "cultural learning" should be considered as "alternative explanations" of behavior, as Zentall's (2016) consideration implies. Any behavior involves a genetic basis, and "basic learning processes" as well as more complex "cultural learning" are no exceptions to this rule
(Lachlan \& Feldman, 2003; Plomin \& Deary, 2015). Moreover, "conditioned responses" may play a role even in complex learning processes (F. C. Davis et al., 2009). Thus, there is no contradiction in the supposition that reciprocal altruism can be based on both genetic predispositions and Pavlovian conditioning, as is the case with any other kind of behavior. The occurrence of reciprocity does not imply that basic learning processes are not involved, but importantly, the reciprocal cooperation observed in rats cannot merely be explained by Pavlovian conditioning, for the following reasons:

1. Zentall (2016) highlights two important experimental preconditions for the conclusion that reciprocal cooperation as observed in Norway rats cannot be simply explained by Pavlovian conditioning. First, when experimental subjects receive experience with a defecting (i.e. noncooperating) partner, they should receive the same amount of food during this experience phase as when they obtain experience with a cooperating partner. This condition was fulfilled in our experiment ("During each non-cooperator session, the experimenter also baited the tray eight times with one oat flake on the side of the focal rat"; Rutte \& Taborsky, 2008, p. 501), hence the rats received the same amount of food during their cooperator and noncooperator experience. Second, it needs to be shown that the food donations of focal test animals indeed reflect a social behavior. Zentall (2016) asks, "Would these rats have pulled the stick even if the other cage had been empty?" This requests an important control that was indeed performed in the cited study:

"One day after this experiment, we noted the pulling rate of each focal rat when alone in the experimental cage to check for intrinsic differences in pulling frequency. The situation was equivalent to the experiment where the rat could move the platform into the cage by pulling, but she was unable to reach the reward, and no partner was present." (Rutte \& Taborsky, 2008, pp. 502-503)

This control revealed that

the baseline pulling frequency recorded 1 day after the experiment when rats were alone in the cage was lower than in the direct reciprocity treatment (Mann-Whitney $\mathrm{U}$ test, $p=0.001)$ and in the generalized reciprocity treatment (Mann-Whitney U test, $p=0.002$ ), respectively, but it did not differ significantly between the treatments. (Rutte \& Taborsky, 2008, p. 503)

Therefore, there is no indication that the intrinsic tendency to pull had been influenced by the experimental treatments. Furthermore, in another study of 
generalized reciprocity (Rutte \& Taborsky, 2007), the experimental subjects were also tested when alone in the cage: After the training of the rats and before starting the experiment, the pulling rates of 20 rats were compared between a situation in which they were alone in the cage and a situation in which their respective partners were present. The pulling rate was significantly higher when the partner was present than when the second compartment was empty. One day after the experiment, the experimental subjects were again allowed to pull food for an empty cage to check for intrinsic differences in pulling frequency. The baseline pulling frequency when alone in the cage was once again lower than in the test phases of the helper and the nonhelper treatments, respectively, and it did not differ between treatments (Rutte \& Taborsky, 2007, p. 1422). Together these results show that the pulling behavior is not simply a "generalization from the first experience," as assumed by Zentall (2016, p. 8), but clearly influenced by the social situation. Furthermore, the intrinsic tendency to pull (i.e., when the test rats were alone in the cage) was not influenced by the experimental treatments (i.e. without potential receiver, the rats did not pull more often after receiving help than after receiving no help in both the direct and generalized reciprocity treatments).

2. In the study referred to by Zentall (2016) that revealed rats to dissociate their social partners with respect to the quality of their help (Dolivo \& Taborsky, 2015a), it was shown that

the differential reciprocation of focal individuals to cooperators providing high- or low-quality food did not decrease between test days 1 and 2, even if the general propensity to pull declined. Differentiation would be predicted to remain similar or to decline with time if mere copying was responsible for reciprocal help, but instead it increased on the second test day, despite no additional benefits or information being obtained from their experimental partners in between. (Dolivo \& Taborsky, 2015a, p. 4).

To make a Pavlovian conditioned response disappear (by extinction), the idea is to present the conditioned stimulus alone (which would be the cooperator or the banana provider according to Zentall's (2016) hypothesis), without the presence of the unconditioned stimulus (which would be the reward according to this hypothesis). This exactly was the case in the reported experiment when focal individuals had the opportunity to provide their partners with food on two consecutive test days. In the case of a Pavlovian association, we would not expect the distinction between cooperators to increase without any additional input received from cooperators, but rather to decrease. More complex mechanisms than Pavlovian conditioning are thus required to explain these findings at the proximate level.

3. In the same study (Dolivo \& Taborsky, 2015a), the focal rats were presented simultaneously with both cooperators and obtained at the same time preferred pieces of banana from one cooperator and the same number of pieces of less preferred carrot from another cooperator. In these conditions, with a pure "Pavlovian mechanism" rats would have globally associated both cooperators with "banana and carrots." However, they were able to distinguish in the test which rat had previously provided which treat to them. In addition, as the cages differed between the experience phase and the test phase (three compartments in the experience phase and two compartments in the test phase), no confounding spatial or other effects that are independent of the social partners' identity could be responsible for the observed results. The focal rats' behavior revealed that they specifically recognized the individual partners that had provided banana from the individuals that had provided carrot, also in a new spatial configuration and on a different day.

Furthermore, simple Pavlovian association explains neither the observation that potential benefits for the recipient of a charitable act can affect the amount of help a donor will provide (Schneeberger, Dietz, \& Taborsky, 2012), nor the finding that reciprocity and coercion may both enhance cooperation in rats if not applied simultaneously (Dolivo \& Taborsky, 2015b).

Thus, rats engage in reciprocal altruism in a way that cannot be simply explained by "a genetic predisposition," or "basic instrumental and Pavlovian processes" (Zentall 2016, p. 8). Apparently, the underlying mechanisms are more complex. There is no "either-or" when considering genetic and phenotypic causes of behavior and other biological traits; understanding behavior at both levels requires an integrative approach (Hofmann et al., 2014; Taborsky \& Taborsky, 2015). This poses a splendid challenge for future studies of reciprocal cooperation of rats.

One cannot overemphasize that the experiments so far performed to investigate reciprocal cooperation in rats do not allow making any assumptions about the motivation leading rats to engage in reciprocal altruism. It is neither justified to attribute their cooperative behavior to the application of some moral principle such as "do unto others as you would have them do unto you," nor to the opposite view pretending that moral principles are surely not involved when rats act according to direct reciprocity 
rules, even if the latter possibility might seem more parsimonious and hence more likely.

Acknowledgments M. T. has been supported by the Swiss National Science Foundation (Project 31003A_156152).

\section{References}

Babb, S. J., \& Crystal, J. D. (2006). Episodic-like memory in the rat. Current Biology, 16, 1317-1321.

Boeckle, M., \& Bugnyar, T. (2012). Long-term memory for affiliates in ravens. Current Biology, 22, 801-806.

Bruck, J. N. (2013). Decades-long social memory in bottlenose dolphins. Proceedings of the Royal Society B: Biological Sciences, 280, 20131726.

Carter, G. G., \& Wilkinson, G. S. (2013). Food sharing in vampire bats: Reciprocal help predicts donations more than relatedness or harassment. Proceedings of the Royal Society B: Biological Sciences, 280, 20122573.

Carter, G. G., \& Wilkinson, G. S. (2015). Social benefits of non-kin food sharing by female vampire bats. Proceedings of the Royal Society B: Biological Sciences, 282, 20152524.

Carter, G. G., \& Wilkinson, G. S. (2016). Common vampire bat contact calls attract past food-sharing partners. Animal Behaviour, 116, 45-51.

Cheney, D. L. (2011). Extent and limits of cooperation in animals. PNAS, 108(Suppl. 2), 10902-10909.

Cockburn, A. (1998). Evolution of helping behavior in cooperatively breeding birds. Annual Review of Ecology and Systematics, 29, 141-177.

Davis, F. C., Johnstone, T., Mazzulla, E. C., Oler, J. A., \& Whalen, P. J. (2009). Regional response differences across the human amygdaloid complex during social conditioning. Cerebral Cortex, 20, 612-621.

Davis, R. L. (2005). Olfactory memory formation in Drosophila: From molecular to systems neuroscience. Annual Review of Neuroscience, 28, 275-302.

de Silva, S., \& Wittemyer, G. (2012). A comparison of social organization in Asian elephants and African savannah elephants. International Journal of Primatology, 33, 1125-1141.

Dolivo, V., \& Taborsky, M. (2015a). Norway rats reciprocate help according to the quality of help they received. Biology Letter, 11, 20140959.

Dolivo, V., \& Taborsky, M. (2015b). Cooperation among Norway rats: The importance of visual cues for reciprocal cooperation and the role of coercion. Ethology, 121, 1071-1080.

Godard, R. (1991). Long-term memory of individual neighbors in a migratory songbird. Nature, 350, 228-229.

Grueter, C. C., Matsuda, I., Zhang, P., \& Zinner, D. (2012). Multilevel societies in primates and other mammals: Introduction to the special issue. International Journal of Primatology, 33, 993-1001.

Hofmann, H. A., Beery, A. K., Blumstein, D. T., Couzin, I. D., Earley, R. L., Hayes, L. D., ... Rubenstein, D. R. (2014). An evolutionary framework for studying mechanisms of social behavior. Trends in Ecological Evolution, 29, 581-589.

Jungwirth, A., \& Taborsky, M. (2015). First- and second-order sociality determine survival and reproduction in cooperative cichlids. Proceedings of the Royal Society B: Biological Sciences, 282, 1819.

Kawecki, T. J. (2010). Evolutionary ecology of learning: Insights from fruit flies. Poputlation Ecology, 52, 15-25.

Kendrick, K. M., da Costa, A. P., Leigh, A. E., Hinton, M. R., \& Peirce, J. W. (2001). Sheep don't forget a face. Nature, 414, 165-166.

Lachlan, R. F., \& Feldman, M. W. (2003). Evolution of cultural communication systems: The coevolution of cultural signals and genes encoding learning preferences. Journal of Evolutionary Biology, 16, 1084-1095.

Mery, F., \& Kawecki, T. J. (2002). Experimental evolution of learning ability in fruit flies. Proceedings of the National Academy of Sciences of the U.S.A, 99, 14274-14279.

Plomin, R., \& Deary, I. J. (2015). Genetics and intelligence differences: Five special findings. Molecular Psychiatry, 20, 98-108.

Riehl, C. (2013). Evolutionary routes to non-kin cooperative breeding in birds. Proceedings of the Royal Society B: Biological Sciences, 280, 1772.

Rutte, C., \& Taborsky, M. (2007). Generalized reciprocity in rats. PLoS Biology, 5(7), 1421-1425. doi:10.1371/journal.pbio.0050196

Rutte, C., \& Taborsky, M. (2008). The influence of social experience on cooperative behavior of rats (Rattus norvegicus): Direct vs. generalized reciprocity. Behavioral Ecology and Sociobiology, 62, 499-505.

Schneeberger, K., Dietz, M., \& Taborsky, M. (2012). Reciprocal cooperation between unrelated rats depends on cost to donor and benefit to recipient. BMC Evolutionary Biology, 12, 41.

Taborsky, M., Frommen, J. G., \& Riehl, C. (2016). Correlated pay-offs are key to cooperation. Philosophical Transactions of the Royal Society of London B, 371, 20150084.

Taborsky, M., \& Taborsky, B. (2015). Evolution of genetic and physiological mechanisms of cooperative behaviour. Current Opinion in Behavioral Sciences, 6, 132-138.

van Doorn, G. S., \& Taborsky, M. (2012). The evolution of generalized reciprocity on social interaction networks. Evolution, 66(3), 651-664.

Veyrac, A., Allerborn, M., Gros, A., Michon, F., Raguet, L., Kenney, J., ... Ravel, N. (2015). Memory of occasional events in rats: Individual episodic memory profiles, flexibility, and neural substrate. Journal of Neuroscience, 35, 7575-7586.

Wright, J., \& McDonald, P. G. (2016). Bell miners: Kin-selected helping decisions. In W. D. Koenig \& J. L. Dickinson (Eds.), Cooperative breeding in vertebrates: Studies of ecology, evolution, and behavior (pp. 272-293). Cambridge: Cambridge University Press.

Zentall, T. R. (2002). A cognitive behaviorist approach to the study of animal behavior. The Journal of General Psychology, 129(4), 328-363.

Zentall, T. R. (2013). Animals represent the past and the future. Evolutionary Psychology, 11(3), 573-590.

Zentall, T. R. (2016). Reciprocal altruism in rats: Why does it occur? Learning \& Behavior, 44, 7-8. 